\title{
RESPONSE OF LOW FREQUENCY HARVESTING SYSTEMS OF RUBBER UNDER DRIER CLIMATIC CONDITIONS IN SRI LANKA
}

\author{
K.V.V.S. Kudaligama ${ }^{1 *}$, V.H.L.Rodrigo ${ }^{1}$, K.M.E.P. Fernando ${ }^{2}$ and P. A. J. Yapa \\ ${ }^{1}$ Department of Biochemistry and Physiology, Rubber Research Institute, Sri Lanka \\ ${ }^{2}$ Department of Botany, University of Sri Jayewardenepura, Sri Lanka \\ *vskudaligama@yahoo.com, Tel-++94 34 2247426, \\ Fax- ++ 94342247427
}

\begin{abstract}
Escalating cost of production (COP) and inadequate supply of skilled harvesters are among the major issues that natural rubber industry face today. Low frequency harvesting (LFH) systems of which trees are tapped in a lesser frequency than once in two days, are considered to be one of the solutions to overcome these issues. With the focus given to expand rubber in drier climates, the present study was aimed to investigate on the yield response of LFH systems, viz. harvesting trees once in three (d3), four (d4) and six (d6) days in the intermediate zone (IZ) with the stimulation protocols developed for the wet zone (WZ). With the decrease in harvesting frequency in IZ, yield per tree per tapping (GTT) increased, however yield per tree per year (YPT) decreased. No such declines were observed in WZ. Stimulation had no negative impact on percentage dry rubber content in latex (\%DRC) or incidence of Tapping Panel Dryness. Whilst only the S/2 d3 system is acceptable for IZ in the present form, stimulation protocols are to be revised in other LFH systems. Bark consumption was reduced significantly by LFH resulting in several long-term beneficial effects. Effect of stimulation on latex physiology is also discussed.
\end{abstract}

Keywords: Climate change, Ethephon, Hevea, Low frequency harvesting, Rubber

\section{INTRODUCTION}

Being the second largest plantation crop in the country, rubber (Hevea brasiliensis) covers ca. 122,000 ha and produces ca. 129million kg of raw rubber (ref). More importantly, it provides $c a$. 500,000 direct and indirect employments. The total export earnings from raw rubber and end products in 2008 was about Rs. 72 billion (Anon, 2008a, Anon, 2008b).

The rubber plantations in Sri Lankan lie in almost the Wet zone (WZ) of the country and certain regions in the Intermediate zone (IZ). The demand for rubber among the farmers in the IZ is escalating rapidly due to the poor turnover from the cash crops faming in those areas. The IZ, running between the Wet and Dry zones demarcates the area, which receives a mean annual rainfall between 1,270 - 2,540 mm with considerable dry spells (Wjesekera, et al, 2004) whilst the annual rainfall in WZ is over 2,500 mm. During the month of April, temperature in IZ can rise up to about $35^{\circ} \mathrm{C}$, however, that in between late November to mid February period is lower than the rest of the year (Anon, 2009).

A uniformly distributed rainfall with about $1650-3000 \mathrm{~mm}$ per year is known to be ideal for the cultivation of rubber. In the areas with an average temperature in the range of $23-28{ }^{\circ} \mathrm{C}$ and altitude less than $200 \mathrm{~m}$ above sea level, rubber tree performs well in establishing, growing and yielding (Yogaratnam, 2001). High relative humidity ( $c a .80 \%)$ and radiation level ( $c a .2000$ hrs/year) influence the plant growth and yield (Pushpadas \& Karthikakutty Amma, 1980, Rao \& Vijayakumar, 1992). Although IZ receives a considerable amount of rainfall, the un-uniform pattern makes stressful conditions to the trees. Longer dry spells during wintering period create adverse effects on both growth and yield.

Traditionally, the rubber tree is tapped with $\mathrm{S} / 2 \mathrm{~d} 2$ (i.e. half spiral cut tapped once in two days) harvesting system. Tapping once in three days $(\mathrm{S} / 2 \mathrm{~d} 3)$ is recommended in Sri Lanka with $2.5 \%$ 
Ethephon with 4-5 stimulation rounds per year (Nugawela, 2001). Harvesting is considered to be the most important operation in rubber plantations since it determines not only the productivity but also economic life of the tree. It requires greater level of skill and also contributes to ca. 1/3 of the cost of production (COP) in Sri Lanka. In some countries, the share of tapping in the COP is as high as $70 \%$ (Vijayakumar, 2003). The survival of the natural rubbers industry is being threatened by the low prices, spiralling cost of production and inadequate supply of skilled harvesters. With the aim of alleviating some of these problems, planters and scientist turned to explore possibility of adopting LFH systems that reduce the number of harvesters required and ultimately the COP. With the change of tapping frequency from $\mathrm{d} 2$ to $\mathrm{d} 3, \mathrm{~d} 4$ or $\mathrm{d} 6$, labour need for harvesting could be reduced by $33 \%, 50 \%$ and $67 \%$ respectively.

LFH systems are known to be profitable, should over $90 \%$ of the yields given by traditional systems be achieved (Kewi \& Sivakumaran, 1994, Tassi et a,l 1991). It is necessary that, trees have to be stimulated under LFH for achieving comparable yields to that of $\mathrm{d} 2$ frequency. For long term sustainable yields, protocols for LFH systems should be designed considering the genotype (clone), prevalent agro climatic conditions and frequency of exploitation of the tree. On this background, the present study was aimed to investigate the response of $S / 2 d 4$ and $S / 2 d 6$ low frequency tapping system in the Intermediate zone of Sri Lanka.

\section{MATERIALS AND METHODS}

A mature field with widely used Sri Lankan Hevea genotype, i.e. clone RRIC 121, was selected from Pitiakanda Group in Kurunegala districts which falls in the category of IL1a in the IZ. All trees were tapped on BO-1 panel. Harvesting systems were based on half spiral cut length (S/2) and $\mathrm{d} 2, \mathrm{~d} 3, \mathrm{~d} 4$ and $\mathrm{d} 6$ tapping frequencies were imposed with different stimulation protocols (i.e. no stimulation, 2.5\% Ethephon 5 rounds/year, 2.5\% Ethephon 9 rounds/year and 5\%Ethephon 12 rounds/year, respectively). Experiment began in 2008 after an initial trial period. Yield and related parameters were continuously monitored from April, 2008 to March, 2009. Latex flow and incidence of tapping panel dryness were also assessed. Yield and related parameters of RRIC 121 planted in Dartonfield estate in WZ and harvested under d2, d3, d4 and d6 frequencies were taken for comparison. Stimulation protocol of each LFH system was alike in both WZ and IZ.

\section{RESULTS}

Total annual rainfall in IZ and WZ for the reporting period was 1890 and $5226 \mathrm{~mm}$, with 121 and 222 wet days respectively. In IZ, seven months of the year received rainfall lower than the minimum level stipulated as favourable for rubber cultivation. However in WZ, such conditions were as only for two months. Dry period in IZ started from the end of January and continued till May. But in this particular period, considerable amount of rain has fallen during March and April. The minimum rainfall in IZ and WZ was received during the months of February \& January and the maximum in October and May, respectively (Fig. 1). Relative humidity (\%RH) in IZ was lower than the WZ. Average \%RH in IZ and WZ was about 78 and 82\%, respectively (Fig. 2). Intermediate zone experienced minimum and maximum temperatures about 22.7 and $32.1{ }^{\circ} \mathrm{C}$, respectively whilst that in $\mathrm{WZ}$ was 22.5 and $32.2{ }^{\circ} \mathrm{C}$, respectively. During the period of January to March, temperature of the day was around $34{ }^{\circ} \mathrm{C}$. Evaporation in the IZ was higher than that of WZ (Fig. 3). Highest evaporation and shine hours in IZ were recorded during January - March period. 


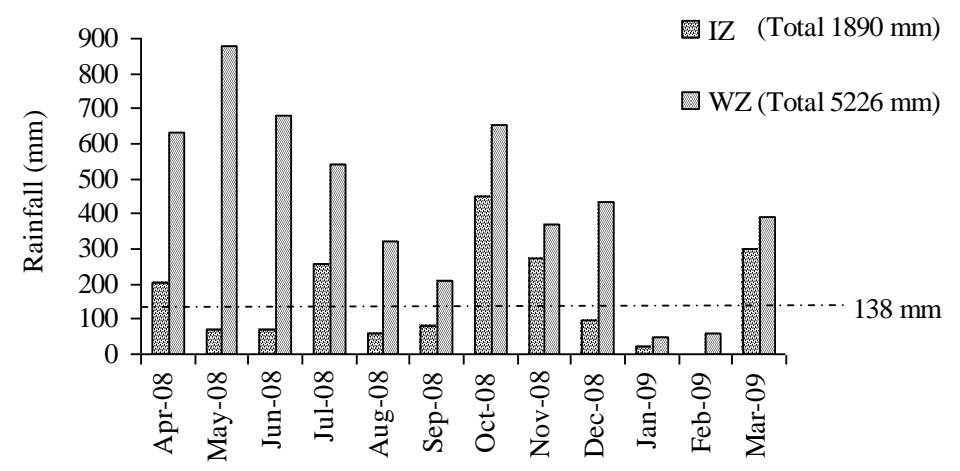

Figure 1: Monthly total rainfall in Intermediate (IZ) and Wet (WZ) zones in Sri Lanka (the minimum expected level for rubber is shown with the dotted line)

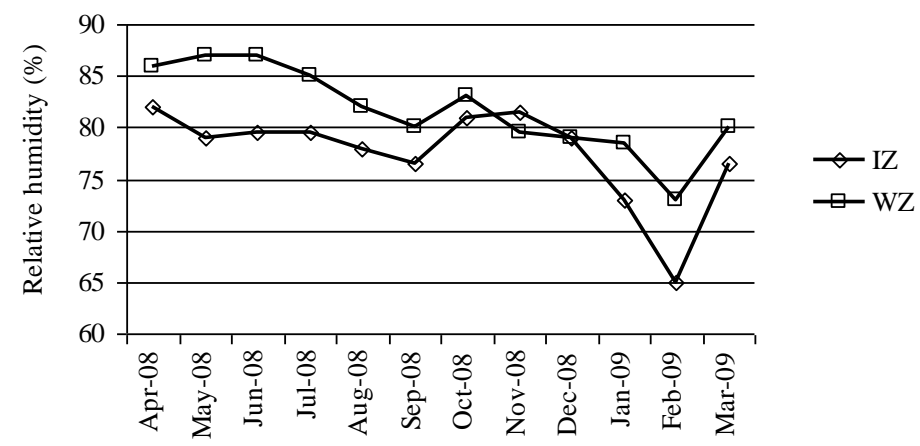

Figure 2: Monthly average relative humidity in Intermediate (IZ) and Wet (WZ) zones in Sri Lanka

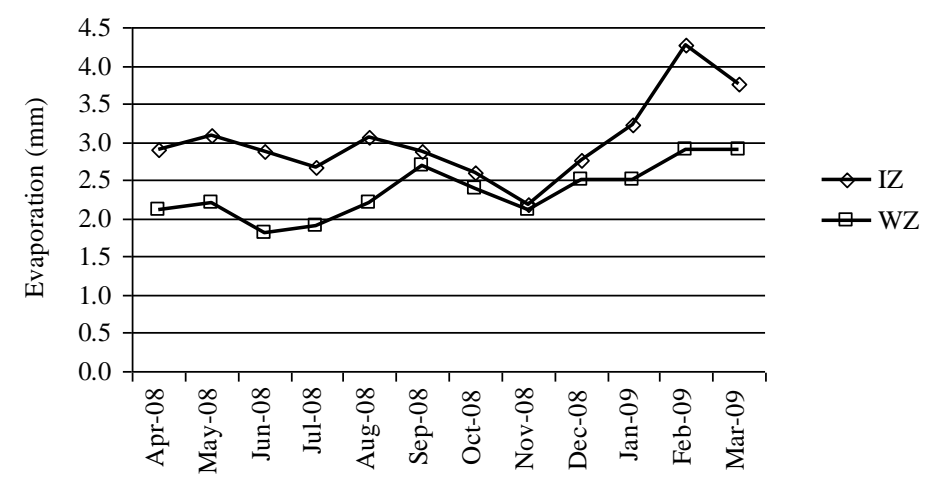

Figure 3: Monthly average evaporation in Intermediate (IZ) and Wet (WZ) zones in Sri Lanka.

Generally, yield per tree per tapping (GTT) increased with the decrease in tapping frequency with the highest values recorded in S/2 d6 tapping system. In both zones, the lowest GTT was experienced during the months of February to March whilst the highest during October to December. During the twelve month period, both $\mathrm{d} 4$ and $\mathrm{d} 6$ frequencies could not provide the expected level of GTT (i.e. 100 and 200\% increase over that in d2, respectively) in IZ (Fig. 4). Therefore, yield per tree per year (YPT) fell off with the decrease in tapping frequency from $\mathrm{d} 2$ to $\mathrm{d} 6$ in the IZ. The $\mathrm{d} 4$ frequency showed a $19 \%$ decline in YPT and it was quite sharp in d6 with $36 \%$ decrease. 

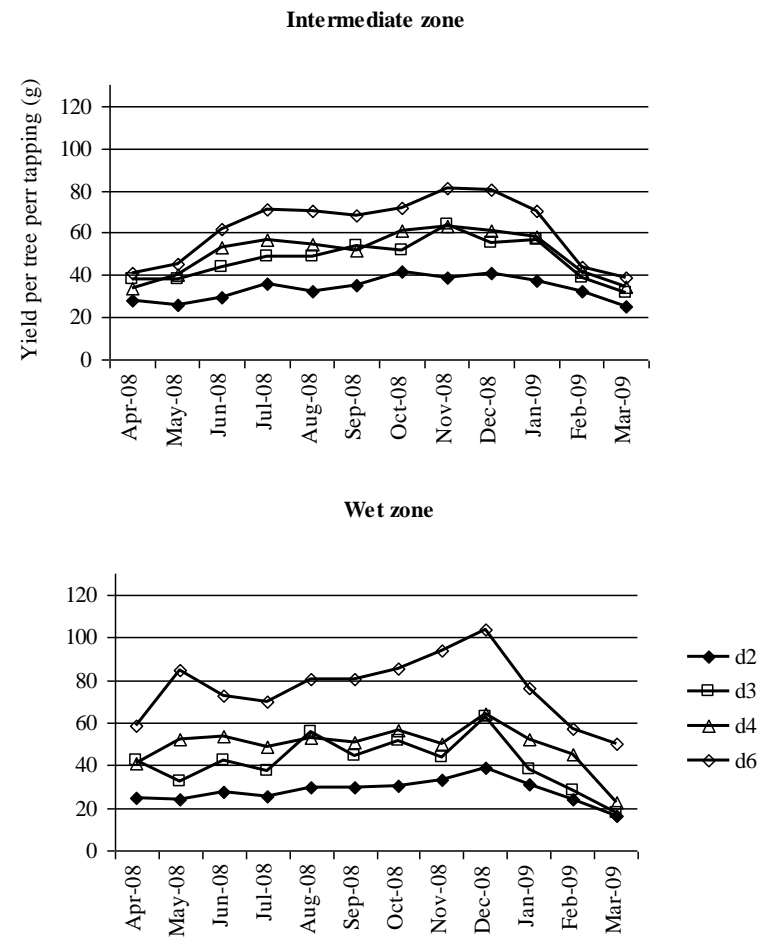

Figure 4: Yield per tree per tapping (GTT) of trees tapped under different harvesting frequencies in Intermediate (IZ) and Wet (WZ) zones of Sri Lanka. Codes d2, d3, d4 and d6 refer to the harvesting frequencies of once in $2,3,4$, and 6 days, respectively

Mean YPT recorded during the year tested was 5.62, 5.49, 4.58 and $3.0 \mathrm{~kg}$ in $\mathrm{d} 2, \mathrm{~d} 3$, d4 and d6 systems, respectively. However, no such decline was observed in WZ with comparable values among all harvesting systems (Fig. 5).

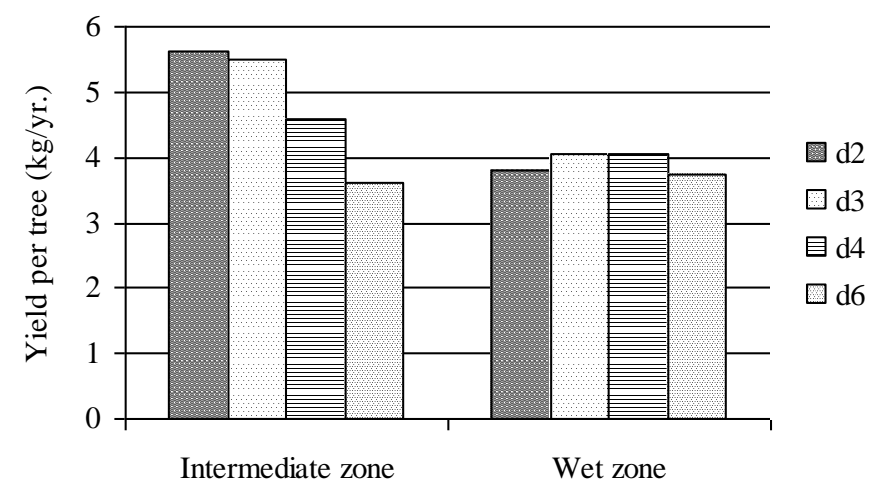

Figure 5: Yield per tree per annum in different harvesting systems. Codes d2, d3, d4 and d6 refer to the harvesting frequencies of once in $2,3,4$, and 6 days, respectively

Percentage dry rubber content in latex (\%DRC) increased with the decrease in the harvesting frequency. In general, \%DRC in WZ was higher than that of IZ in all four harvesting frequencies. Volume of latex extracted increased with the reduction of the harvesting frequency. However, such volume increase in IZ was not as high as in WZ. The volume harvested from $\mathrm{d} 3, \mathrm{~d} 4$ and $\mathrm{d} 6$ frequencies increased only by $36 \%, 47 \%$ and $76 \%$ over $\mathrm{d} 2$ value in IZ whilst that increase in WZ was $42 \%, 68 \%$ and $160 \%$, respectively (Table 1 ). 
Table 1: Average \%dry rubber content and volume of latex harvested under different low frequency systems. Codes $\mathrm{S} / 2 \mathrm{~d} 2, \mathrm{~S} / 2 \mathrm{~d} 3, \mathrm{~S} / 2 \mathrm{~d} 4$ and $\mathrm{S} / 2 \mathrm{~d} 6$ refer to the harvesting frequencies of once in 2,3,4, and 6 days, respectively

\begin{tabular}{lcccc}
\hline Tapping & \multicolumn{2}{c}{ Dry rubber content of latex (\%) } & \multicolumn{2}{c}{ Latex volume (l) } \\
\cline { 2 - 5 } system & Intermediate zone & Wet zone & Intermediate zone & Wet zone \\
\hline $\mathrm{S} / 2 \mathrm{~d} 2$ & 32 & 36 & 29.05 & 22.93 \\
$\mathrm{~S} / 2 \mathrm{~d} 3$ & 34 & 38 & 39.73 & 32.76 \\
$\mathrm{~S} / 2 \mathrm{~d} 4$ & 33 & 38 & 42.85 & 38.99 \\
$\mathrm{~S} / 2 \mathrm{~d} 6$ & 34 & 38 & 51.62 & 59.66 \\
\hline
\end{tabular}

Overall bark consumption in terms of bark consumption per year decreased with the decrease in tapping frequency. Mean values recorded were $37 \%, 47 \%$ and $64 \%$ less in $\mathrm{d} 3$, d4 and d6 tapping frequencies, respectively from the bark consumption in $\mathrm{d} 2$. However, average bark consumption per tapping did not show a distinct variation among the tapping frequencies with a mean value of $0.15 \mathrm{~cm}$ (Table 2).

Table 2: Bark consumption and number of tapping days under different harvesting frequencies Codes are as same as in Table 1.

\begin{tabular}{lccc}
\hline $\begin{array}{l}\text { Tapping } \\
\text { system }\end{array}$ & $\begin{array}{c}\text { No. of tapping } \\
\text { days }\end{array}$ & $\begin{array}{c}\text { Bark consumption } \\
(\mathbf{c m} / \mathbf{y r})\end{array}$ & $\begin{array}{c}\text { Bark consumption per } \\
\text { tapping (cm) }\end{array}$ \\
\hline S/2 d2 & 168 & 24.94 & 0.15 \\
S/2 d3 & 116 & 15.84 & 0.14 \\
S/2 d4 & 90 & 13.32 & 0.15 \\
S/2 d6 & 58 & 9.09 & 0.16 \\
\hline
\end{tabular}

Table 3: Latex flow rate and plugging index under different harvesting frequencies and Codes are as same as in Table 1 .

\begin{tabular}{lcccc}
\hline \multirow{2}{*}{ Tapping system } & \multicolumn{2}{c}{ Flow rate (ml/hr.) } & \multicolumn{2}{c}{ Plugging index } \\
\cline { 2 - 5 } & Intermediate zone & Wet zone & Intermediate zone & Wet zone \\
\hline $\mathrm{S} / 2 \mathrm{~d} 2$ & 70.7 & 35.8 & 3.02 & 5.66 \\
\hline $\mathrm{S} / 2 \mathrm{~d} 3$ & 73.7 & 36.9 & 3.08 & 2.96 \\
\hline $\mathrm{S} / 2 \mathrm{~d} 4$ & 79.0 & 43.3 & 1.49 & 1.97 \\
\hline $\mathrm{S} / 2 \mathrm{~d} 6$ & 84.3 & 52.0 & 1.28 & 1.74 \\
\hline
\end{tabular}

In general, the flow rate increased with the reduction in harvesting frequency in both climatic zones. Percentage change in the flow rate of LFH systems over $\mathrm{d} 2$ frequency was greater in WZ. Plugging index (PI) decreased along with the frequency though PI of d2 and d 3 systems was quite comparable in IZ (Table 3).

\section{DISCUSSION}

Obviously, low frequency harvesting (LIH) systems are capable of reducing workers requirement in latex harvesting hence lowering the cost of tapping which accounts for $1 / 3$ of COP. For instance, $\mathrm{d} 3, \mathrm{~d} 4$ and $\mathrm{d} 6$ tapping reduces the requirement of latex harvesters by 33,50 and $67 \%$ from that of $\mathrm{d} 2$. As shown by GTT, the reduction of harvesting frequency has increased the daily intake which is supposed to compensate the yield loss due to less number of tapping days. In addition to the nominal daily wage, latex harvesters are paid for rubber harvested above the norm; 
hence, increased intakes in LFH increase the incentive payment providing an improved income to the harvester. Of course, prolong latex dripping and increased intakes demand extra energy and time to collect latex and so, justify the need of such additional payments. Cost involved in the application of stimulants (i.e. chemical and labour for application) is another cost component involved in LFH systems. However, both such costs (i.e. incentive payment and stimulation cost) are easily compensated by the cost saving from less number of tapping days in LFH and so in short profits, what is important is to obtain overall yields of LFH comparable to traditional S/2 d2 system. According to Kewi \& Sivakumaran, (1994) and Tasi et a,l (1991), breakeven point for finance lies at about $90 \%$ of the overall yield produced by $S / 2 \mathrm{~d} 2$ and therefore, though all LFH systems are found suitable for WZ, only S/2 d3 system is acceptable for IZ. Nevertheless, with revised stimulation protocols, other systems could also be made effective. Therefore, further analyses are required to find out the exact amount of Ethephon for each frequency and the best day for stimulating (especially for d6 frequency). Further, number of stimulations per year could also be manipulated to achieve the required yield levels.

Overall yield is given by YPT which comprises GTT and number of tapping days. In WZ, all LFH systems tested were able to produce over $90 \%$ of yield that given by d2 frequency though it is not a case in IZ. However in d4 frequency, only $8 \%$ increase is required to achieve the $90 \%$ level; hence only a slight change in its stimulation protocol would be sufficient.

As shown in the present study, reduction in bark consumption is another beneficial effect of LFH. This increases the economical life span of the rubber tree hence, delays the replanting. With the overall adjustment towards the reduction in immature : mature ratio, it has positive impact on the cash flow of rubber estates. Less bark consumption also provides additional time for bark renewal hence better yield could be expected from the renewed bark. As a whole, reduction in replanting cost component, higher yields from renewed bark and environmental benefits resulted from delay in replanting have positive impacts on overall economics sustaining the rubber plantations having $\mathrm{LFH}$ in long run.

Although overall bark consumption was reduced significantly with the reduction in tapping frequency, the rate of its reduction did not exactly proportional with tapping frequency due to the associated increase in the shaving thickness (i.e. bark consumption per tapping).

Values recorded for \%DRC in all harvesting systems tended to decline from January to March. This may be an effect of defoliation/wintering as well as the low rainfall prevailed during this period. Although $\mathrm{d} 2$ frequency showed comparatively lower \%DRC values during rest of the period, \%DRC was more or less similar in all four frequencies during the wintering period. This means drop in \% DRC in LFH is greater during wintering and so, studies are required to assess the stress level to the tree with potential over stimulations.

Ethephon is thought to act as a chemical which enhance latex production and delays plugging of latex vessels (Gomez, 1983; Abraham et al., 1971). Both initial flow rate and duration of flow increased after stimulation in this study as reported before (de Jonge, 1955, Chapman, 1951). This means that a considerable amount of water is extracted from trees in latex harvesting. In particular, such extraction is greater with the reduction in tapping frequency. From February to April, the volume of latex harvested is more or less equal in all low frequency systems. Defoliation and dry spells should be the primary cause, however stimulation under these conditions may create an extra stress to the tree as no increase in latex volume. Latex production is closely dependent on climatic conditions such as rainfall, relative humidity and temperature and in particularly on the availability of water in soil (Yogaratnam, 2001). Reduction in rubber synthesis in adverse conditions is masked by the reduction in latex volume and therefore, \%DRC did not fall below $30 \%$.

Highest number of TPD affected trees were recorded under $\mathrm{d} 2$ frequency hence, there was no indication on increased incidence of TPD affected trees with stimulations. However, in the event 
of increasing the yields of LFH systems in IZ (to be comparable with S/2 d2 yields), detailed studies would be required on its effects on TPD. Particularly during dry seasons and defoliation period negative impact of stimulation has been observed (Abraham \& Tayler, 1967).

\section{CONCLUSION}

Although all LFH systems tested are found suitable for WZ, only S/2 d3 system is acceptable for IZ. Nevertheless, with revised stimulation protocols, other systems could also be made effective. Therefore, further studies are required to find out the exact amount of Ethephon under each frequency and the best day for stimulating (especially for d6 frequency). Further, number of stimulations per year could also be manipulated to achieve the required yield levels. Bark consumption was reduced significantly by LFH resulting in several long-term beneficial effects such as extended productive lifespan, higher yields from renewed bark, reduced replanting needs and altogether economic viability. There was no indication in increased incidence of TPD affected trees in LFH systems tested.

\section{ACKNOWLEDGEMENT}

This study was funded by the National Science Foundation under the contract research programme of RG/2006/AG 07. Valuable assistance given by Mr. P.D.J. Rodrigo, Mr. R. P. S. Randunu and Mr D. S. Hewamanage of the Department of Biochemistry and Physiology of Rubber Research Institute and the staff of Pitiakanda Group of Lalan Rubbers PLC, Sri Lanka are highly appreciated.

\section{REFERENCES}

Abraham, P. D., Blencowe, J. W., Chua, S. E., Gomez, J. B., Moir, G. F. J., Pakianathan, S. W., Sekhar, B. C., Southorn, W. A., Wycherley, P. R., 1971. Noval stimulants and procedures in the exploitation of Hevea. I. Introductory review. Journal of Rubber Research Institute of Malaya, 23, 85.

Abraham, P. D., Tayler, R. S., 1967. Stimulation of latex flow in Hevea brasiliensis, Exp. Agr. 3, 1.

Ahmad Zarin Mat Tasi, Kewi, Chong, Hashim, Ismail, 1991. Low intensity tapping systems and early use of CUT. In: Towards Greater Viability of the Natural Rubber Industry. Rubber Growers' Conference, Kuala Lumpur, Malaysia July 1991. 189-211.

Anon, 2008a. Statistical information on plantation crops, Ministry of Plantation Industries, Colombo, Sri Lanka.

Anon, 2008b. Annual report of Central Bank of Sri Lanka.

Anon, 2009. Sri Lanka Business Directory, Kurunegala District, North Western province of Sri Lanka. http://www.buyandsell.lk/north-western/kurunegala/index.html\%20-\%20 [Accessed on 15 May 2010].

Chapman, G. W., 1951. Plant hormones and yield in Hevea brasiliensis, Journal of Rubber Research Institute of Malaya, 13, 167.

De Jonge, P., 1955. Stimulation of yield in Hevea brasiliensis, III Further observations on the effects of yield stimulation, Journal of Rubber Research Institute of Malaya, 14, 383.

Gomez, J. B., 1983. Physiology of latex (rubber) production, Rubber Research and Development Board of Malaysia, Monograph No 8.

Proceedings of the $15^{\text {th }}$ International Forestry and Environment Symposium, 26-27 November 2010.

Published by Department of Forestry and Environmental Science, University of Sri Jayewardenepura, Sri Lanka. 
Kewi, Chong, Sivakumaran, S., 1994. Performance of low frequency tapping systems. Workshop of the exploitation technologies to address current labour problems in the rubber industry, Kuala Lumpur, Malaysia. 47.

Nugawela, A., 2001. Exploitation for economic yields. In: L. M. K. Tillekerathne, A Nugawela (Eds.), Handbook of Rubber Volume 1, Rubber Research Institute of Sri Lanka, Agalawatta, Sri Lanka, 176-190.

Pushpadas, M. V., Karthikakutty Amma, M., 1980. Agro-ecological requirements. In: Radhakrishnan Pillay, P. N. (Eds.), Hand book of natural rubber production in India, Rubber Research Institute of India, Kottayam, India. $87-109$.

Rao, P. S., Vijayakumar, K. R., 1992. Climatic requirements. In: Sethuraj, M. R., Mathew, N. M. (Eds.), Natural rubber biology, cultivation and technology, Rubber Research Institute of India, Kottayam, India, 220-238.

Vijayakumar, K R, 2003. Low frequency tapping to reduce cost of natural rubber production. Indian Rubber Journal, 70, 41.

Wijesekera, R. S., Rajapakse, R. A. G. R. R., Somasiri, L. L. W., Tennakoon, N. A., 2004. Hydrological study on deep groundwater aquifers in the coconut growing areas of Kurunegala District. Water Professionals' Symposium, Colombo, Sri Lanka, October, 2004. Available from: http://www.gissl.lk/WaterProf/2004/acrobatpapers/RS_Wijesekera_18.pdf [Accessed on 15 May 2010]

Yogarathnam, N., 2001. Land suitability evaluation, selection and soil conservation. In: Tillekeratne L. M. K., Nugawela A. (Eds.), Hand book of rubber, Agronomy, Rubber Research Institute of Sri Lanka. 